Simon D. Angus (Clayton)

Monika J. Piotrowska (Warszawa)

\title{
THE ONSET OF NECROSIS IN A 3D CELLULAR AUTOMATON MODEL OF EMT6 MULTI-CELLULAR SPHEROIDS
}

Abstract. A 3-dimensional (3D) extension to a previously reported scaled 2-dimensional Cellular Automaton (CA) model of avascular multi-cellular spheroid growth is presented and analysed for the EMT6/Ro cell line. The model outputs are found to compare favourably with reported experimentally obtained data for in vitro spheroids of the same cell line. Necrosis (unprogrammed central cell death) is observed to be delayed when compared with the experimental data. Furthermore, it is found that necrosis arises in the model due to subcritical nutrient conditions and not due to toxicity (modelled as the production of $\mathrm{H}^{+}$ions) as suggested by some authors. Indeed, central $\mathrm{pH}$ conditions, which can be followed in a facile manner in the CA setup, are never observed to reach critical levels. Implications of these results are considered both for the $\mathrm{CA}$ model approach and the underlying understanding of tumour metabolism and progression.

1. Introduction. Recently it has been realized that cells cultivated in 3 -dimensional (3D) contexts behave differently from those studied as monolayers (see e.g. [14]). Hence, it can be argued that a 3D modelling context is an important element of effective tissue modelling. Indeed, in the present study, we study a mathematical model that follows a widely used in vitro experimental approach in which multicellular spheroids (MCS), i.e. spherical aggregates of cells, are grown and studied. It should be noted that in such experiments biologists are only able to investigate the mechanisms gov-

2010 Mathematics Subject Classification: 92C37, 92-08.

Key words and phrases: 3-dimensional cellular automaton, multicellular spheroid, cell metabolism, necrosis. 
erning the tumour development in the first stage of neoplastic disease, i.e. the avascular tumour growth phase.

In the modelling literature there exist several cellular automata (CA) and agent based $(\mathrm{AB})$ models in which a $3 \mathrm{D}$ approach has been attempted (see e.g. [10], [11] or [23]). Here we extend our previously reported 2D CA model ([17], [18]) to the 3D CA context. In the present approach the basic cellular metabolism and proliferation are described on the mesoscopic (cell) level and this allows us to investigate the macroscopic properties of growing tumours at the tissue level. In particular, rather than employ the abstraction of dimensionless concentration and length units, we seek to fully scale the model space so that experimentally observable features are accessible to the inquirer. This approach not only affords the opportunity to bring the model to conformity with currently available experimental data, but enables predictions for future experimental observations to be confirmed or otherwise.

Our goal was to investigate the onset and progression of necrosis within the in vitro cultivated MCS. Our main finding is that necrosis (unprogrammed cell death) appears in the central region of the model tumour due to the subcritical concentration of nutrients $(\mathrm{CHO})$ that arises from the reaction-diffusion dynamics of the system. It is to be noted that this finding occurs despite the inclusion of a plausible waste (acid) producing metabolism scenario for cells in the developing tumour model. That is, the results of the present work in 3D concur with those of our earlier work in 2D ([17]) that waste products modelled only as acidic metabolic by-products are not sufficient to induce necrosis at the appreciably earlier times observed in vitro. Thus, we use the results of this model to identify some requirements for additional modelling elements that are required to reproduce the results of in vitro studies with higher fidelity. As part of our study, we also extend the peripheral mitotic growth theory to account for the bulk dynamics of such CA approaches and to motivate our novel many-to-one scaling assumption that makes feasible the modelling of large (approaching clinically observable sizes) tumour spheroids.

This paper is organized as follows: Section 2 introduces the peripheral mitotic growth theory for the 3D CA, Section 3 contains the detailed description of the proposed CA model, while in Section 4 the main numerical results are presented and compared with the experimental data. Finally, in Section 5 these results are discussed with particular reference to the necrotic process together with a description of future directions for this research.

2. Peripheral mitotic growth \& the CA approach. We shall investigate a 3D CA model on a regular orthogonal lattice (see Fig. 1, right). To investigate the growth mechanism of the $\mathrm{CA}$ approach, we propose an 

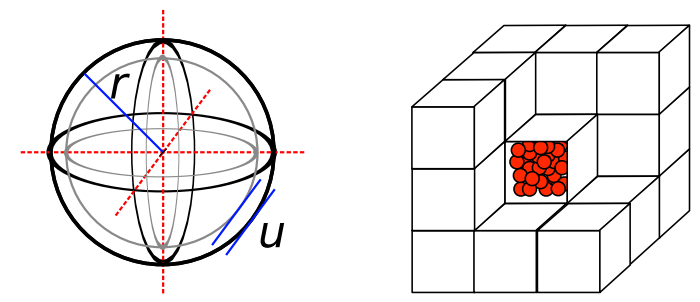

Fig. 1. Geometry of the 3D space, showing radius, $r$, and proliferative surface interpretation of the site side-length, $u$ (left); implementation of many-to-one assumption in the cut-away 3D lattice (right)

extension of the 2D peripheral mitotic growth theory (presented in an earlier work, [17, [18]) to the 3D case. Namely, we shall assume that the mitotic region of the tumour is concentrated at the periphery of the tumour mass. Indeed, although not widely acknowledged in the literature, the CA approach forces this assumption since any inner tumour mass site that is completely surrounded by filled sites will be restricted from generating a daughter site nearby. It should also be noted that the one-to-one assumption carries with it the implication that the peripheral boundary is of only one $C A$ site thickness. For a standard one-to-one setup, this would imply that the proliferating region is of thickness close to the diameter of a single cell, namely, around $10 \mu \mathrm{m}$. Or in other terms, it is assumed by the one-to-one assumption that a cell/site at just two sites (or about $20 \mu \mathrm{m}$ ) inside the surface of the growing tumour mass would be unable to produce a daughter cell nearby due to contact inhibition.

In contrast, our approach relaxes the one-to-one assumption by assuming a many-to-one approach where any number $N$ of cells can be considered to reside in a single CA site. Not only does this approach allow for the peripheral mitotic region to expand to realistic widths (on the order of $100 \mu \mathrm{m},[21,5]$ ), but also it allows the effective study of very much larger tumour masses (up to the scale of $1 \times 10^{6}$ cells) whilst keeping the number of computational elements within implementable margins (e.g. on the order of $\left.1 \times 10^{4}\right)$. Clearly, one loses a degree of detail in the results as a consequence of this approach, since all cells inhabiting a single site are effectively treated as a homogeneous packet.

We can progress this discussion by formalising the implications of the many-to-one assumption on bulk tumour properties. We shall focus on the MCS growth before the necrosis in the middle of the tumour occurs. Thus, we consider a proliferating boundary of thickness equivalent to the side-length of one CA site (refer to Fig. 1, left), i.e. thickness $u$ in the figure. Under this assumption, we may consider the following equation:

$$
\frac{d C(t)}{d t}=k N \epsilon(t)
$$


for the rate of tumour cell count $(C(t))$ progression at time $t$, where $k$ is the rate constant (units $t^{-1}$ ), $N$ is the fixed number of cells per $C A$ site as defined above (units cells per site) and $\epsilon(t)$ is the number of $C A$ sites at the proliferation boundary of the tumour mass at time $t$ (units sites).

We note that the sites of proliferation now sit on the entire surface of the sphere, and hence we obtain

$$
\epsilon(t)=4 \pi\left(\frac{r(t)}{u}\right)^{2}
$$

for the expected number of sites at the surface (of course, assuming the CA tumour mass can be approximated by a smooth sphere of radius $r$ at time $t$ ). Similarly, assuming that the total number of CA sites is equal to the ratio of total idealised sphere volume to the volume of one CA site we obtain for the total cell count in the developing tumour,

$$
C(t)=\frac{4}{3} \pi N\left(\frac{r(t)}{u}\right)^{3} .
$$

After rearrangement and substitution we can rewrite (2.1) as the following separable dynamic equation:

$$
\frac{d C(t)}{d t}=k(4 \pi N)^{1 / 3}(3 C(t))^{2 / 3} .
$$

The general solution to this system is easily found to be

$$
C(t)=\left[\left(\frac{4}{3} \pi N\right)^{1 / 3} k t+C^{1 / 3}(0)\right]^{3} .
$$

Hence, taking into account the fact that $u^{3}=N / \rho$, where $\rho$ is the cells packing density, one obtains the following formula for the diameter progression with time:

$$
d(t)=2 k(N / \rho)^{1 / 3} t+d(0),
$$

where $d(0)=2(3 C(0) / 4 \pi \rho)^{1 / 3}$ is the initial MCS diameter. This scheme recovers the familiar linear relationship between diameter and time of growth (2.6), and suggests a cubic relationship between cell count and time (2.5). Importantly, this theory indicates that the scaling assumption made concerning the number of cells per site $(N)$ in the CA setup can be used to rescale any particular data into a regime of a different scale. This suggests that the scaling assumption does not interfere with the bulk tumour properties in any meaningful way.

3. The MCS model. The present 3D approach is a natural extension of previous results for a $2 \mathrm{D}$ model reported in [17]. Indeed the model analysed in this work follows almost identically in setup, scaling and calibration with only the dimensionality of the context differing between the two works. Thus, the reader is referred to the more complete description of the model setup 
in the reference. However, some minor modifications and extensions to these elements have been made and will be described below.

We shall briefly review the main elements of this setup. First, the model geometry used is 3D in nature (refer to Fig. 1). The "world" is divided into equally spaced, square lattice sites in which it is envisaged that the tumour cells sit. We assume no cellular "migration" to or from nutrient sources (as is assumed in e.g. [12]), instead occupied tumour sites may only become unoccupied due to cell-death, or propagate to an adjacent neighbouring site via cell division.

Second, the key many-to-one assumption of the previous work has been retained. As in [17] the CA lattice sites are assumed to be unit-cubes containing $N$ cells. The value of side-length $u$ is calculated analogously to [17] by taking into account the cell packing density $(\rho)$ which gives $u=(N / \rho)^{1 / 3}$ in $\mu m$. Thus, the choice of $N$ becomes the key scaling parameter in this setup. As elaborated in [17] the choice of $N$ is guided by the value of the resulting site side-length $u$. This is because in a strict CA approach as we describe in this work the majority of division events will occur at sites at the periphery of the tumour (since sites at the periphery are guaranteed to have free adjacent sites available for placing daughter cells into). Hence, we can say that $u$ is also the approximate thickness of the peripheral cell rim, which according to [21, 5] is of the order of $100 \mu \mathrm{m}$. For the model to follow, $N$ is thus set to a constant value of 400 such that $u$ obtains the length $100 \mu \mathrm{m}$.

It is assumed that the model spheroid grows in a well-mixed, constant concentration substrate laced with glucose and oxygen at $n_{e x}$ and $o_{e x}$ respectively. The $\mathrm{pH}$ of the substrate is also held constant at 7.4. This assumption is justified by the periodic replenishment of substrate reported in the standard experimental method (e.g. [5]). We note that although our "world" is only a factor of approximately two times greater than the spheroidal size, in reality, this factor would be at least two orders of magnitude larger, and hence the consumption of the tumour cells would have little effect on the substrate concentrations under periodic replenishment.

Each site undergoes a basic cellular metabolism process involving the uptake of nutrients in the form of oxygen and glucose and the production of $\mathrm{H}^{+}$ions. There are various approaches to this metabolism reported in the literature (see e.g. [16], [20] or [8]). In the present approach, we have employed a somewhat simplified approach compared to some of the more complex metabolism models available. Following [17] we assume that sites may exist in one of five states: aerobic proliferation, anaerobic proliferation, aerobic quiescence, anaerobic quiescence, and finally, site death. It is assumed in the model that cells (proliferating and quiescent) living in insufficient oxygen conditions perform anaerobic metabolisms which, since it is less efficient 
than the aerobic metabolism, forces hypoxic cells to consume much more glucose when compared to cells living in an oxygen-rich environment.

The determination of which state a site is in at any time-step is determined by the state transition algorithm which is reproduced from [17] in Fig. 2 below. The values of critical levels of glucose, oxygen and $\mathrm{pH}$ that determine each step in the algorithm follow identically those reported in Table 1 of [17]. Note that in this work, as in our previously reported 2D approach, there is no differentiation between programmed (apoptosis) and unprogrammed (necrosis) site death, instead, it is assumed that each site death is of an unprogrammed (necrotic) nature.

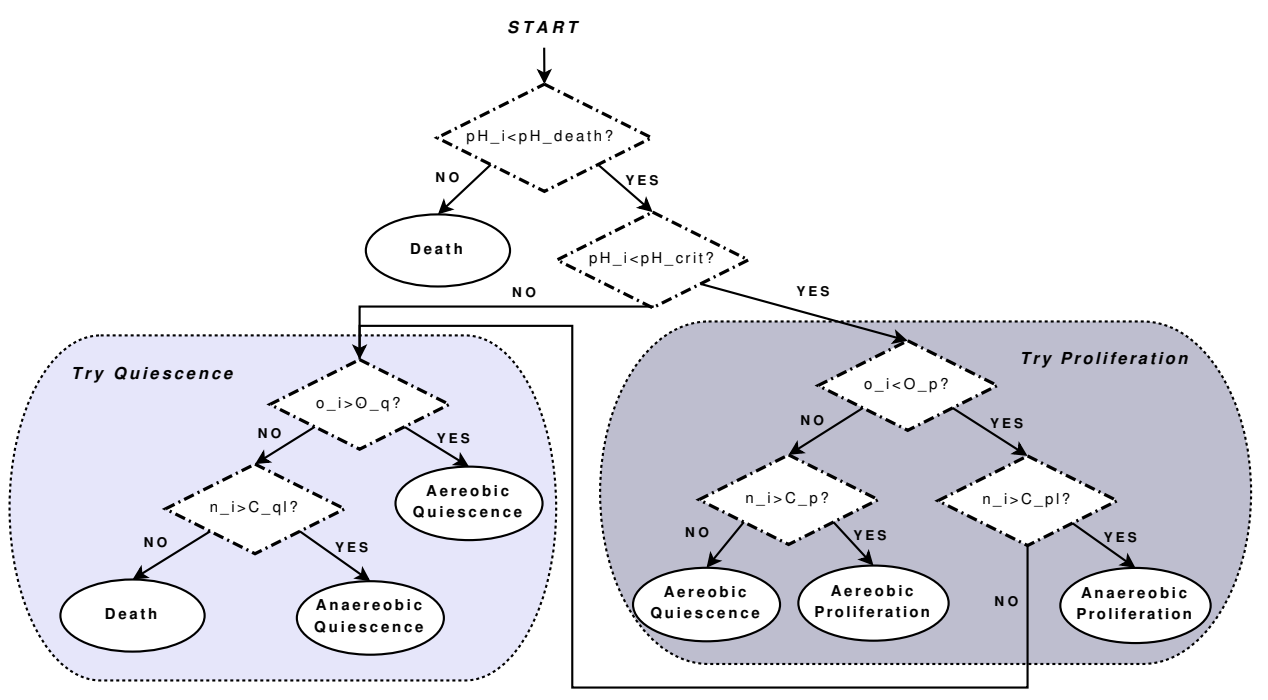

Fig. 2. The state selection algorithm Update site states applied to each site following 17

Due to the discretized nature of the CA world, a numerical diffusion algorithm is applied within the tumour to mimic the diffusion of nutrients from the external solution into the tumour mass. To accomplish the internal diffusion we track the nutrients concentrations $\left(\mathrm{CHO}\right.$ and $\left.\mathrm{O}_{2}\right)$ as well as $\mathrm{pH}$ for all lattice sites. The diffusion process can be represented for site $i$ as

$$
x_{i}^{t+1}=x_{i}^{t}+\alpha\left(\frac{1}{18} \sum_{j \in \mathcal{N}^{i}} x_{j}^{t}-x_{i}^{t}\right),
$$

where $x_{i}^{t}$ is the quantity of nutrients at site $i$ at time $t$ and $\mathcal{N}^{i}$ is the 18-member neighbourhood surrounding site $i$ (diagonal corners removed). A numerical diffusion coefficient $\alpha$ stands in for the standard diffusion coefficient and is equal to $D_{n}, D_{o}$ and $D_{H^{+}}$for glucose, oxygen and acid, 
respectively. Section 3 below details the determination of the values of the numerical diffusion coefficients.

A relevant update in this work is to recalculate the probabilities for diffusion and cell-division on the 3D lattice to ensure isotropy of growth and nutrient transfer (i.e. to overcome square or diamond morphologies as observed in e.g. [22] or [9]). One can attempt to naturally extend the 2D algorithm reported for the $2 \mathrm{D}$ case in [17]. However, after some algebra, it is apparent that an isotropic solution does not exist on a square lattice when all 26 adjacent sites are included as potential diffusion or division neighbours. However, an isotropic solution is obtainable if the problem is reduced to only the 18 von Neumann type neighbours; that is, without the diagonal corners included (Fig. 3). If one defines the speed of progression

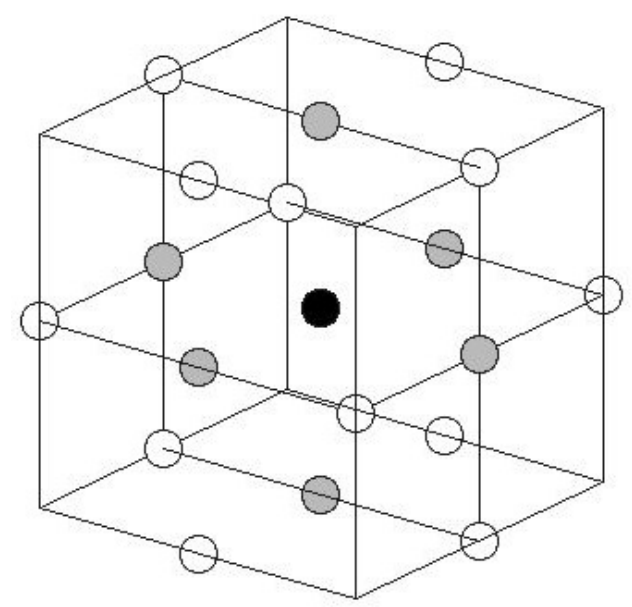

Fig. 3. The reduced neighbourhood used to ensure isotropy for the discrete numerical diffusion algorithm and the daughter cell placement algorithm. The black site indicates the centre of the cube with sites at the faces (grey) and first-order diagonals (white) also shown.

in the $x$ axis (orthogonal to the face, i.e. the grey points in the figure) as $e_{x}$ and the same in the direction of a first-diagonal (the edge points, i.e. the white points in the figure) as $e_{x y}$, and the probability of placing a new element at a face position and edge position as $p$ and $q$ respectively, then one obtains the simultaneous equation system

$$
\begin{aligned}
e_{x} & =1 p+4 q, \\
e_{x y} & =\sqrt{2} p+2 \sqrt{2} q,
\end{aligned}
$$

which for $e_{x}=e_{x y}=1$ has solution $p^{*}=0.4142$ and $q^{*}=0.1464$. Utilisation of these calculated values of $p^{*}$ and $q^{*}$ gives the spherical tumour morphology as desired. 
With the model geometry as outlined above all experimentally measured parameters such as consumption rates, medium glucose concentration etc. are rescaled based on the site size that arises after the choice of the scaling parameter $N$.

An overview of the timing of the present model is given in Algorithm 1. After scaling and initialisation, the main loop is entered, running until the specified experiment time $T$ has been reached (set to 19 days in the results that follow). As noted above, the external (medium) nutrient concentrations of glucose and oxygen are first updated to simulate spheroid growth in a large, well mixed vessel. The numerical diffusion algorithm then works on only the subset of sites which are occupied by cells, which is followed by nutrient accumulation by each site. This step mimics the osmotic transfer across the cell membrane.

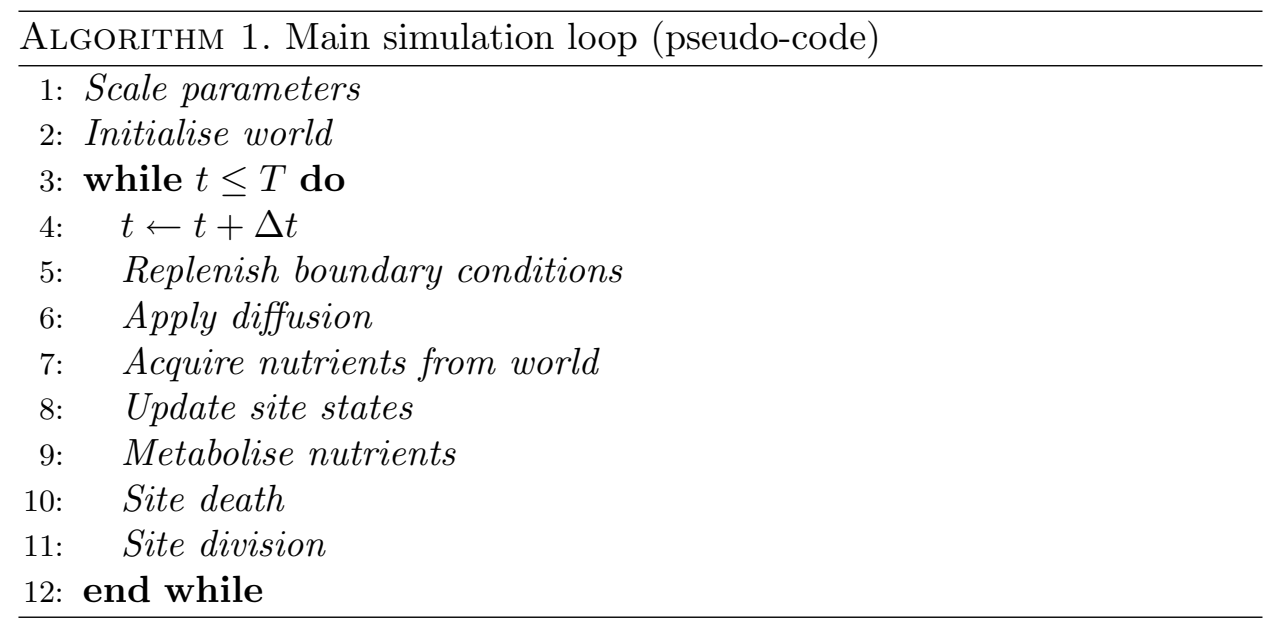

Next, given the levels of nutrients obtained by each site during the nutrient acquisition step, all sites have their state updated, which guides the metabolism actions in the following step. For instance, sites in the aerobic proliferation state will consume $C_{p}$ and $C_{o}$ from their glucose and oxygen stores respectively, whilst also updating their mitotic register by one time step. (Other actions can be compared with the data given in Table 1 in [17.) Site-death is then enacted for those sites in the death state, which in the present setup means that the site becomes immediately vacant.

Finally, mitosis (site division) is handled in our setup by initialising all lattice sites (cell packages) with their own, unique mitotic register, $p_{i}$. This register is advanced by $\Delta t$ for any time-step that the site spends in one of the two proliferative (aerobic or anaerobic) state regimes. In this way, it is assumed that proliferative sites are always preparing for a mitotic event. Moreover, each site is endowed with its specific site-cycle (mitotic) time at 
"site-birth" (when a packet of cells is placed in the site) which is chosen from a normally distributed population $\bar{p} \sim N\left(p_{0}, \sigma_{0}\right)$, where $p_{0}$ is the average cell cycle time and $\sigma_{0}$ is the standard deviation. The normal distribution has been chosen for each site's cycle-time for two reasons. First, genetic differences between cells contribute to variation in cycle times that theoretically and experimentally appear to fit this distribution, [19], [15, and second, this variation helps to overcome a numerical artefact that could arise with constant cycle times, namely, an unnatural "step" growth phenomenon (all sites proliferating in synchronicity). The lattice sites which have accrued their own cycle-time in the proliferation register $p_{i}$ become candidates for division. Hence, mitosis occurs for any sites that have accumulated at least their specific cell cycle time and have a free neighbour site in their 18 neighbours. It should be mentioned here that during the division the whole new package of $N$ homogeneous cells appears in the system.

Diffusion coefficient calibration. Due to the difficulties of matching reported diffusion coefficients to those of the cellular automata 3D setup, a calibrative approach had to be used. In this sense, the value of the numerical diffusion coefficient was the key control parameter of the model. The approach taken was to calibrate the glucose diffusion coefficient such that the diameter growth rate for given glucose and oxygen substrate concentrations matched closely the reported diameter growth rate from [5] for the first 7 days of the experiment before the necrosis in the middle of the growing tumour occurs. The diffusion coefficients for oxygen and acidic waste were then calculated from the resulting glucose numerical diffusion coefficient according to the experimentally reported ratios of oxygen and waste (acid) to glucose diffusion as reported in the literature.

Table 1. A comparison of diffusion coefficients available in the literature, scaled values and the calibrated values as used is in the model. See text for details.

\begin{tabular}{c|cc|cc}
\hline $\begin{array}{c}\text { Diffused } \\
\text { substance }\end{array}$ & $\begin{array}{c}\text { Literature } \\
\text { value }\end{array}$ & Source & $\begin{array}{c}\text { Direct } \\
\text { scaling } \\
\text { value } \\
\left(\mathrm{cm}^{2} \mathrm{~s}^{-1}\right)\end{array}$ & $\begin{array}{c}\text { Calibrated } \\
\text { numerical } \\
\text { value }\end{array}$ \\
$\left(\mathrm{u}^{2} . \Delta t^{-1}\right)$
\end{tabular}

The calibration study was carried out at the target glucose and oxygen substrate concentrations of $5.5 \mathrm{mM}$ and $0.28 \mathrm{mM}$, respectively with 10 trials conducted over a 7 -day time-line with systematically varying numerical diffusion coefficients in $\{0.1,0.2, \ldots, 0.5\}$. A linear least squares fit was then obtained to the pooled diameter data for each condition to obtain a 
calibration curve for the daily diameter growth against numerical diffusion coefficient. Following [5] a desired diameter growth rate of $75 \mu \mathrm{m} /$ day was read off from this calibration curve (using the interp1 procedure in MAT$\mathrm{LAB}$ ) and gave a numerical glucose coefficient of 0.176. Correspondingly, the diffusion coefficients for oxygen and waste (acid) were obtained by a simple ratio calculation from the literature values and came to 0.035 and 0.0212 , respectively (see Table 1 for a comparison).

4. Results. In this section we present the main results of the simulation experiments performed with the MATLAB programming language and with an identical numerical simulation set-up to that of our previous 2D experiments (see Table 1 in [17]) with the exception of the newly calibrated numerical diffusion coefficient (procedure described above) $\left({ }^{1}\right)$.

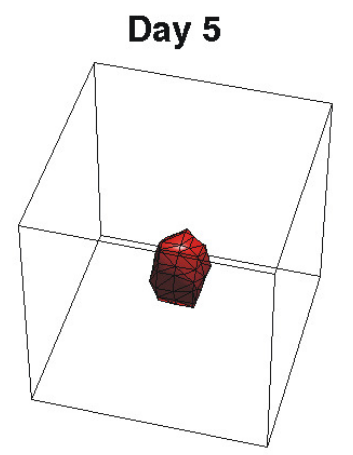

Day 14

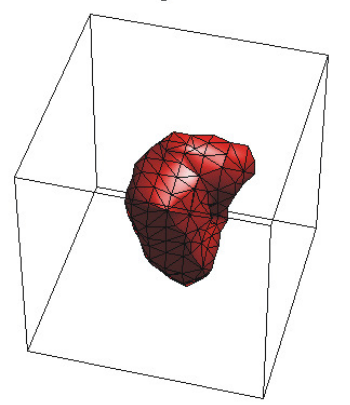

Day 10

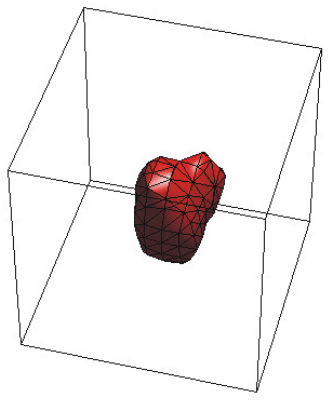

Day 19

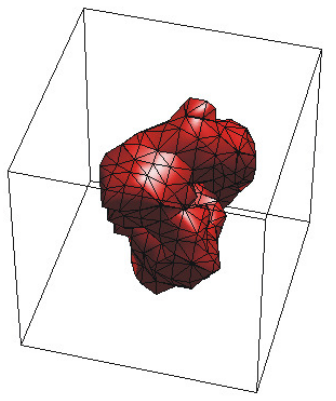

Fig. 4. Example growing tumour mass visualisation for $n_{e x}=5.5 \mathrm{mM}$. Surface represents result of applying smooth3 and isosurface procedures in MATLAB. Lighting and colours (see pdf file) are given as an aid to the reader only. Square box has side-length approx. $1400 \mu \mathrm{m}$ in each case.

$\left({ }^{1}\right)$ It is to be noted that Table 1 of [17 incorrectly reported the value of $N$ used for the experiments, $N=400$, as used in this work. All other parameters are correctly reported. 
Since we shall be largely comparing the results of our study to those of [5], we will similarly consider the effects of changes in the external/substrate glucose concentration, whilst focussing (unless otherwise stated) on the case where $n_{e x}=5.5 \mathrm{mM}$ and $o_{e x}=0.28 \mathrm{mM}$ as in [5] and [6]. To orientate the reader, we present in Fig. 4 a visualisation of a characteristic tumour evolution over the 19-day experiment. As can be seen in the figure, the tumour's final morphology is guided to some extent by the result of stochastic placements of daughter cells after division at early time steps. For instance, the small nodule apparent in the right middle-ground of the tumour at Day 10 in the figure is seen to mature further by Day 14 and Day 19 into a larger growth.

Next we present in Fig. 5 an example progression of key bulk tumour characteristics, again for the $n_{e x}=5.5 \mathrm{mM}$ case. Pleasingly, each trial shows close adherence to the expected linear diameter growth pattern over time (Fig. 5(a)). Similarly, the Gompertz model of total cell count $C(t)$, given by

$$
C(t)=C_{0} \exp \left[\frac{A}{B}(1-\exp (-B t))\right]
$$

is seen to fit the simulation data extremely well (Fig. 5(b)).

For comparison, several external glucose concentrations were studied with the model while all other medium substance concentrations were fixed. Results from the curve fitting exercise to these data are given in Table 2 ,

As expected, the external glucose concentration has little systematic effect on either the initial diameter $\theta_{0}$ or diameter growth rate $\alpha$ results. By definition of the cellular automata spheroid, growth is concentrated at the peripheral sites which always confront the substrate concentration of nutrients and in each case studied is more than enough to sustain proliferation. The values for $\theta_{0}$ are all a little larger than the actual $100 \mu \mathrm{m}$ that the seed site would give but this is understandable given that the model spheroid takes some time to approximate a spherical object. After the calibration as described, the diameter growth rates are all close to the $75 \mu \mathrm{m}$ recorded by Freyer and Sutherland ([5]) for the same tumour cell line (see Table 2 ).

The model initial doubling times appear to be positively related to external glucose concentration which is somewhat counter-intuitive; one should expect that higher nutrient concentrations would lead to faster early growth rates and so lower initial doubling times. However, it should be noted that these results are from the non-linear Gompertz fitting procedure and are sensitive to fitting methods and early data where in the case of the model spheroids the early time-periods correspond to when the model least accurately conforms to a true "spheroidal" shape. For comparison, experimental data under the same conditions as reported in [6] show initial doubling times varying in the range $23-26$ hours with no discernible relationship to glucose 


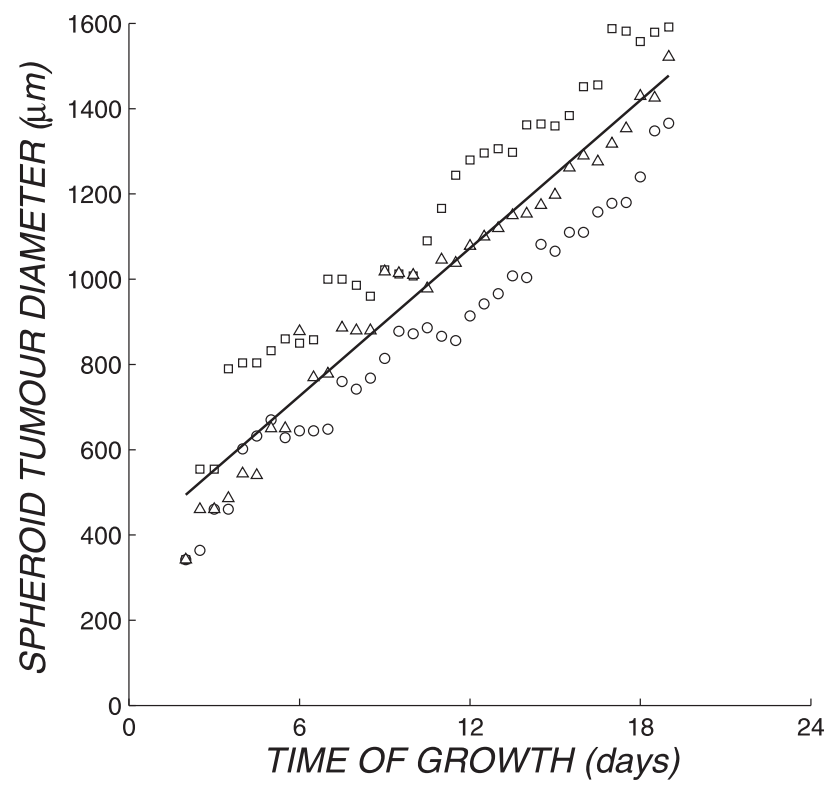

(a)

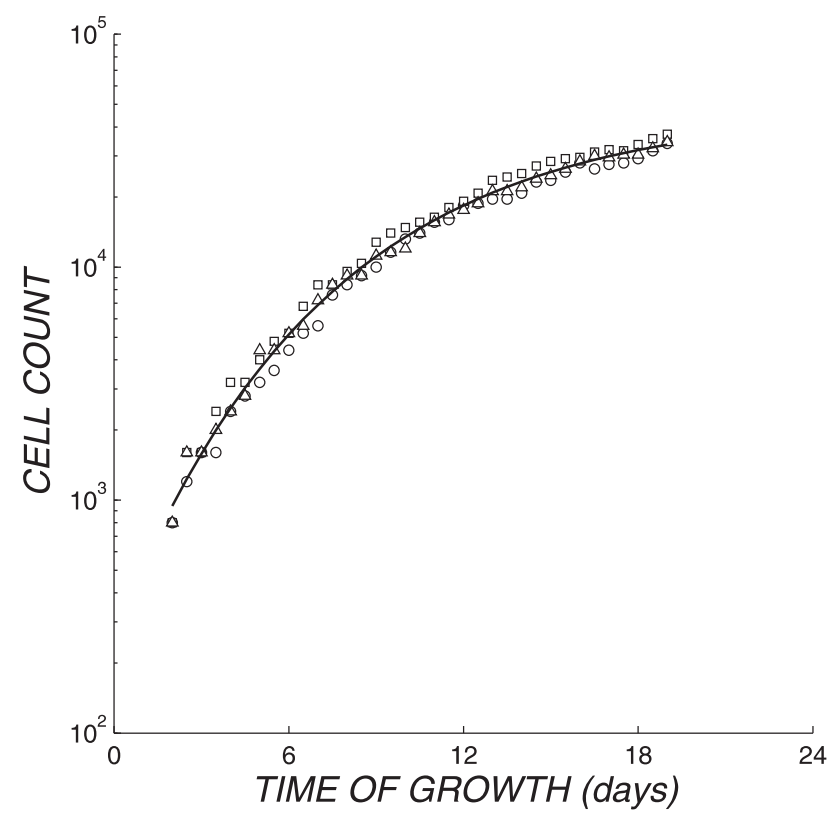

(b)

Fig. 5. Diameter (a) and cell count (b) progression of model tumour with time of growth for $n_{e x}=5.5 \mathrm{mM}$. Results from three experiments are shown with different symbols, the lines of best fit are to all data. (a) Linear fit has slope $57.9 \mu \mathrm{m} /$ day and intercept $379 \mu \mathrm{m}$; (b) Gompertz non-linear fit has coefficients $C(0)=261.7, A=0.735$ and $B=0.141$. 
substrate concentration. A direct comparison of experimental and model doubling times under equivalent conditions yields a close match with glucose concentrations of $5.5 \mathrm{mM}$ and $16.5 \mathrm{mM}$ in [6] giving initial doubling times of 23 and 26 hours respectively where the model gave 22.6 and 30.1 hours (see col. 7, Table 2).

Table 2. Coefficients resulting from curve fitting to diameter and cell count data as for Fig. 5 (medium $n_{o x}=0.28 \mathrm{mM}$ and $p H=7.4$ ).

\begin{tabular}{|c|c|c|c|c|c|c|c|c|}
\hline & \multicolumn{2}{|c|}{ Diameter $^{a}$} & \multicolumn{5}{|c|}{ Cell count ${ }^{b}$} & \multirow{3}{*}{$\begin{array}{l}\text { Thickness } \\
\text { of viable } \\
\text { cell rim }^{d} \\
\quad(\mu \mathrm{m})\end{array}$} \\
\hline$n_{e x}$ & $\theta_{0}$ & $\alpha$ & $C_{0}$ & $A$ & $B$ & $\begin{array}{c}\text { Initial } \\
\text { dbl. time }\end{array}$ & $C(\infty)^{c}$ & \\
\hline$(\mathrm{mM})$ & $(\mu \mathrm{m})$ & $(\mu \mathrm{m} /$ day $)$ & & & & (hrs) & $\times 10^{-4}$ & \\
\hline 2.8 & 310.4 & 65.7 & 261.1 & 0.808 & 0.202 & 20.6 & 1.43 & 323.4 \\
\hline 5.5 & 378.7 & 57.9 & 261.7 & 0.735 & 0.141 & 22.6 & 4.80 & 358.2 \\
\hline 8.0 & 396.0 & 54.6 & 326.2 & 0.626 & 0.115 & 26.6 & 7.44 & 387.3 \\
\hline 10.0 & 330.8 & 57.1 & 360.4 & 0.568 & 0.100 & 29.3 & 10.35 & 474.2 \\
\hline 13.0 & 394.9 & 51.3 & 374.3 & 0.555 & 0.097 & 30.0 & 11.21 & na \\
\hline 16.5 & 365.8 & 58.7 & 375.1 & 0.553 & 0.097 & 30.1 & 11.36 & na \\
\hline
\end{tabular}

Notes

${ }^{a}$ The linear diameter versus time model $\theta(t)=\theta_{0}+\alpha t$ was estimated by OLS to pooled data from all trials for a given substrate glucose concentration experiment.

${ }^{b}$ Cell count versus time data was estimated by the fminsearch procedure in MATLAB to fit the standard Gompertz model $C(t)=C_{0} \exp \left[\frac{A}{B}(1-\exp (-B t))\right]$.

${ }^{c}$ Saturation cell count computed from fitted Gompertz coefficients.

${ }^{d}$ Thickness of viable cell rim determined as mean thickness of cell rim after onset of necrosis, defined as the point at which necrotic volume fraction was larger than $1 \%$; 'na' indicates insufficient necrosis observed to justify measurement.

Of interest to the literature is the relationship between the saturation spheroid cell count and the substrate concentration. The CA is a facile system to obtain such results, which are presented in tabular form in the penultimate column of Table 2 and graphically in Fig. 6. For ease of comparison with experimental data obtained under the same conditions, data from Freyer and Sutherland's 1986 study ([6]) are also shown in the figure. However, although qualitatively the present study produces accurately the overall shape of this relationship, quantitively it does not. Recalling that the substrate conditions for the model were $n_{e x}=5.5 \mathrm{mM}$ and $o_{e x}=0.28 \mathrm{mM}$ it can be seen that the equivalent saturation cell counts reported in [6] (closed squares) are approximately an order of magnitude larger. Indeed, the present study appears to match closely the experimental condition of $o_{e x}=0.07 \mathrm{mM}$ studied by [6] (open squares in the figure). This finding indicates that further work is required to adequately calibrate experimentally determined diffusion coefficients to numerical diffusion coefficients used in our mod- 


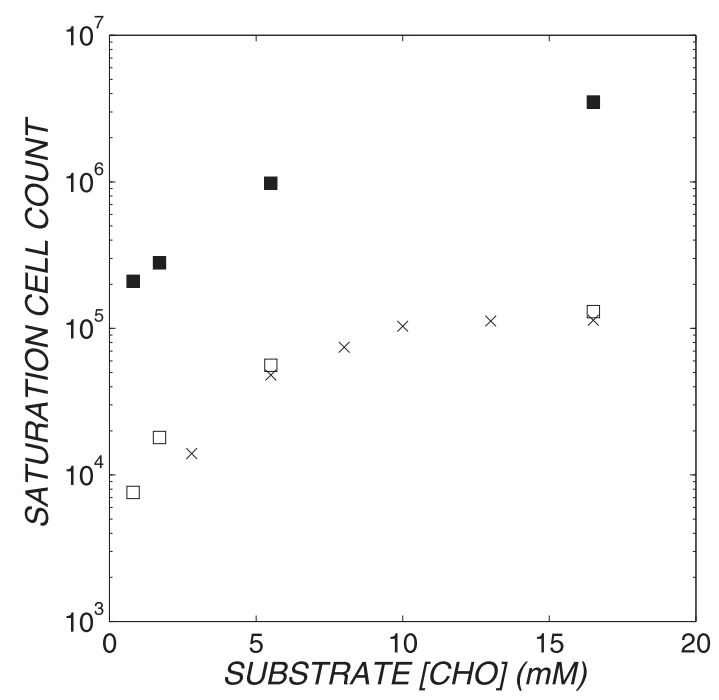

Fig. 6. The relationship between saturation cell count (Gompertz method) and substrate glucose concentration. Mean model data $(\times$, average of three trials) is compared with experiment data for $\left[O_{2}\right]=0.28 \mathrm{mM}(\mathbf{\square})$ and $\left[O_{2}\right]=0.07 \mathrm{mM}(\square)$ as reported in 6 ] (Table 2, p. 3507).

elling approach. Nonetheless, given that the error in this case appears to be something of a simple $y$-shift with the shape of the experimental functional relationship accurately recovered by the model, one can be confident that further calibration should narrow the quantitative model-experiment gap.

The final column of Table 2 reports the relationship between external glucose concentration and viable cell rim thickness. On the whole, it would appear that the model thickness values are larger than those of the experimental tumours. For instance, 3] reports a viable cell rim after necrosis of $251 \mu \mathrm{m}\left([\mathrm{CHO}]=5.5 \mathrm{mM},\left[\mathrm{O}_{2}\right]=0.28 \mathrm{mM}\right)$, or [6] has the same as $203 \mu \mathrm{m}$ whereas the model value was $358.2 \mu \mathrm{m}$. It should be noted that this measurement is sensitive to the definition of "necrosis", with the present work considering the moment of onset of "necrosis" to be when the necrotic volume fraction is larger than 1\%. For instance, if instead the value of $10 \%$ is used as the definition of necrosis onset, the mean viable thickness measurements become $277 \mu \mathrm{m}$ and $285 \mu \mathrm{m}$ for substrate glucose concentrations of $2.8 \mathrm{mM}$ and $5.5 \mathrm{mM}$, respectively. Nevertheless, the measured thickness is significantly larger than that of the experimental tumours.

5. Discussion. One of the key features of interest in the literature is the onset and progression of necrosis $([13,5,6])$. Our previous work with a $2 \mathrm{D}$ version of the present model ([17]) drew two conclusions regarding necrosis: first, that necrosis did not occur in the model due to toxic conditions 
resulting from anaerobic metabolism (as suggested, for example by [6]), but rather necrosis occurred due to a critical glucose concentration, which was reached in the centre of the tumour; and second, that when necrosis did occur, it appeared later in the model tumour than in the experimental literature. Perhaps unsurprisingly, the present work utilising a 3D version of the model concludes similarly.

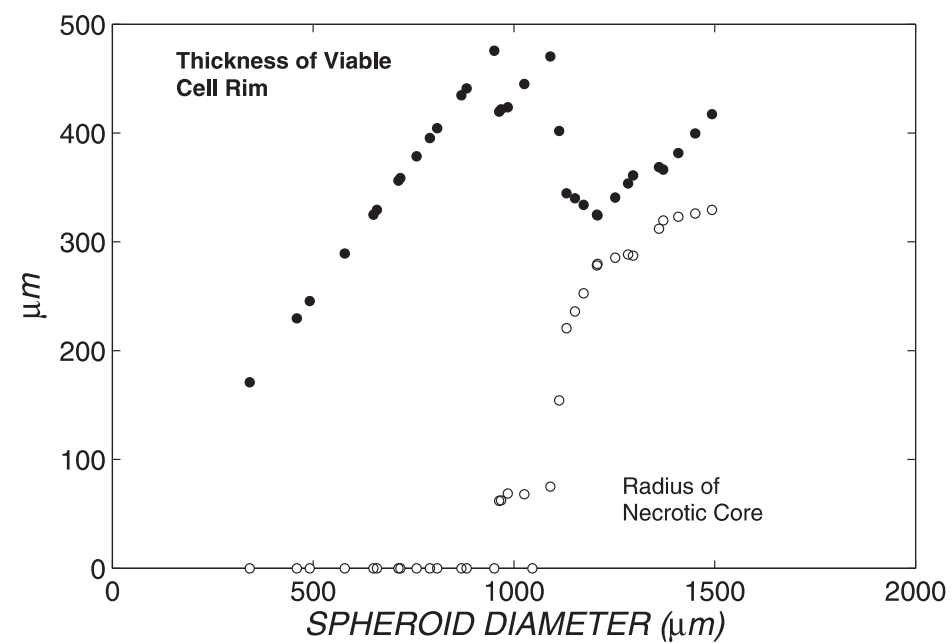

Fig. 7. The onset and progression of necrosis for $n_{e x}=5.5 \mathrm{mM}$. Each point represents mean of three trials at stated conditions.

The first finding is again supported by considering the evolution of the central nutrient and acid concentrations as reported in Figs. 7 and 8 (compare Fig. 10 in [17, though note $n_{e x}=16.5 \mathrm{mM}$ for the plot in [17]). Fig. 7 shows a qualitatively sound progression of necrosis after onset at tumour diameter of around $1000 \mu \mathrm{m}$ (compare Table 3 (p. 520) in [5]) with the viable cell rim progressing as half the spheroid diameter up until the point of necrosis followed by a relatively sudden onset of depletion then stabilisation of the viable cell rim progression.

In the central nutrient plot in Fig. 8 the vertical line represents the point at which necrosis was initiated in the tumour (defined as the necrotic volume fraction being larger than 1\%). The plot is given with normalised concentrations on the $y$-axis such that the upper-limit represents the external (substrate) concentration each substance, whilst the lower-limit represents the critical concentration at which the cell death program would be initiated in the model. As can be seen, whilst the toxicity of the central environment does increase in concert with the increasingly hypoxic conditions, both concentrations of oxygen and acid do not come close to their critical cell-death values. Indeed, necrosis appears in the model simultane- 


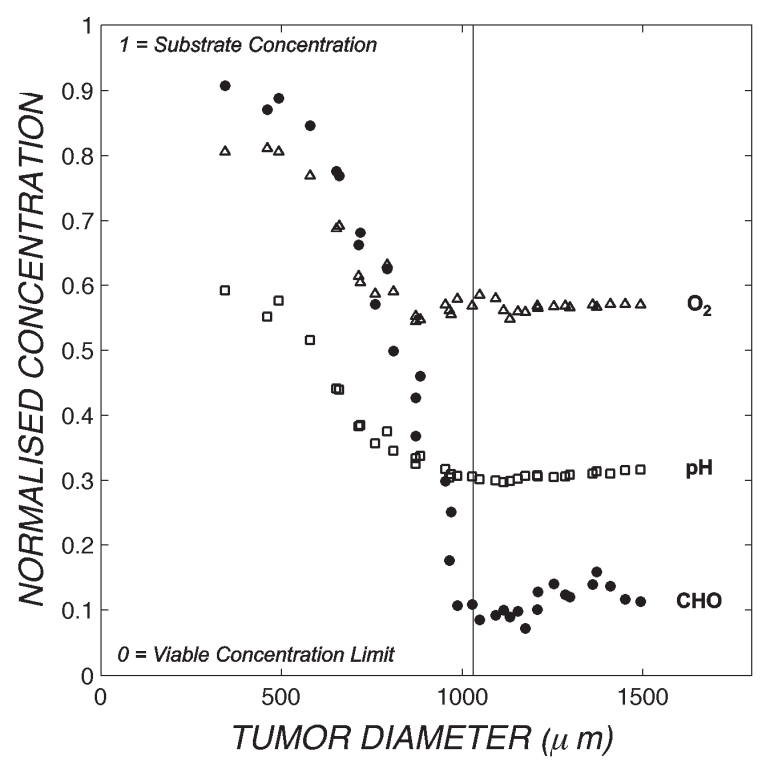

Fig. 8. Mean central concentrations of glucose, oxygen and $\mathrm{H}^{+}$for simulated spheroids at $n_{e x}=5.5 \mathrm{mM}$. The $y$-axis has been normalised so that unity represents the substrate concentration whilst zero represents the concentration at which the site would initiate death due to critically low nutrient concentration (glucose, oxygen) or toxicity ( $\mathrm{pH}$ ).

ously with the approach of the central glucose concentration to within $10 \%$ of its critical value. The values reported in the figure are mean data from a $300 \mu \mathrm{m}$ cube (i.e. the central 27 sites) at the centre of the tumour so allow for subcritical concentrations within this area whilst the mean stays supercritical but sufficiently close to critical. These data can be compared with those obtained by bioluminescence imaging reported in [21] where, as in the figure, oxygen depleted suddenly to its minimal value, followed later by glucose approaching its minimal central value.

Furthermore, as can be seen on the $x$-axis of Fig. 8 , necrosis begins at a tumour diameter of approximately $1000 \mu \mathrm{m}$. This value compares with $413 \mu \mathrm{m}$ and $468 \mu \mathrm{m}$ as reported in [10] and [5] respectively for EMT6/Ro spheroids under equivalent conditions. Indeed, the delay in the onset of necrosis reported here for the 3D model is far greater than that reported in our previous work where necrosis appeared for the same conditions at a tumour diameter of $500 \mu \mathrm{m}$. This delay explains other anomalous data as reported above such as the size of the viable cell rim after the onset of necrosis (see last column in Table 2) which, as reported above, appears to be approximately twice the size of the experimentally determined viable cell rim length. If necrosis appears in the centre of the tumour when the tumour is almost double the size of the experimental tumour, the viable cell rim is 
likely also to be approximately double the size of the experimental cell rim throughout the progression of necrosis.

6. Conclusions. The present study has extended our previous work ([17]) from the 2D to the 3D modelling context. In particular, modelling challenges such as retaining isotropy in the $3 \mathrm{D}$ context and diffusion coefficient calibration have been handled in our approach to yield relatively accurate bulk tumour growth characteristics. As with our previous efforts, the cellular-automaton model presented here is based on the key many-to-one assumption which enables the study of tumour characteristics over a wide diameter scale. The present work corroborates previous indications derived from the 2D model that (a) the onset of necrosis occurs appreciably later in the model setup than is observed in the experimental laboratory with real spheroids; and (b) necrosis arises in the model due to a lack of nutrients (specifically glucose) in the centre of the tumour and not due to high levels of toxicity due to acid waste products.

Hence, these findings support those of our earlier work and suggest at least two directions for further inquiry. First, it is clear that deficiencies in the model setup could be solely to blame for the discrepancy between the model and experimental tumours, and to this end, we address such concerns below; and second, even if the model were to be improved in calibration or parametrisation, it may still suggest the same result, which would indicate that the fundamental theoretical basis of the model is wanting. Dealing with this latter consideration presently, we note that the concept of "waste" perhaps deserves further reflection in the literature. For instance, the elegant experimental study of Freyer ([3]), where extract from the necrotic core of spheroids was diluted and used as the substrate for subsequent spheroid growth, reported marked decrease in doubling time, total number of cells, and clogogenic efficiency with increased exposure to, or concentration of, the spheroid extract substance. Clearly, the inner spheroid material is at least inhibitory, if not toxic, to the viable cells of the spheroid. However, the present study has shown that if this extract (or by-product of cell metabolism) is modelled as $\mathrm{H}^{+}$ions, the central $\mathrm{pH}$ at the time of necrosis cannot be the trigger for the onset of central cell death.

Addressing this issue in the original paper ([3]), Freyer points out that the source of the toxic components in the extract could be from one of at least three sources: by-products of metabolism (perhaps hypoxic metabolism); by-products of the necrosis events themselves; or a reaction of by-products from metabolism or necrosis with the local environment or the viable cells that produces a further toxic compound. Taking the 2D ([17]) and 3D results (this work) of our model together, we can tentatively suggest that the second and third mechanisms proposed by Freyer cannot be wholly responsible for 
necrotic activity, at least not the onset of necrosis. The reason for this is that both of these latter mechanisms rely on a positive feedback theory that takes necrotic action as the cause of further necrosis. However, the present work shows that the first stage of necrosis - its onset, and its further progression, are most likely to occur due to subcritical nutrient levels, not to high localised toxicity. We refer to the near zero slope of the central $\mathrm{pH}$ data shown in Fig. 8 as support.

This discussion thus focusses attention on the basic metabolism driving the cellular activity, and as such, the underpinning setup of the model. On this front, the present approach shows promise. Several qualitative, and some quantitative properties, of spheroid growth in the model match the experimental evidence but the delay in the onset of necrosis is the most stark feature that demands further adjustment. To this end, we are presently reviewing the metabolism module of our setup with a view to including the genetic phases of the cell cycle (G1, S, G2, and M phases) to enable close inspection of the metabolism component with reference to the literature. For instance, data presented in [5] (Fig. 6) and [7] (various figures) show the progression of G1, S and G2 + M phase cells with tumour diameter at different concentrations. Such data would enable close scrutiny of the model's metabolism which is presently unavailable. Furthermore, we are aware that the waste metabolism processes of tumour cells is an area of active research. A revisitation of this aspect of the model could also be helpful (see discussion on this point in [17]). Nevertheless, for the reasons outlined already above, we believe that an acid-induced necrotic initiation is an unlikely outcome of such a revision, given (for example) the present distance from critical levels that central $\mathrm{pH}$ obtains (see Fig. 8).

Finally, the present calibrated approach to the numerical diffusion coefficients would ideally be revisited in future work, yielding a truly scaled approach as utilised for all other parameters. However, we are not at present aware of a suitable approach to this problem. As mentioned, the reported values for diffusion coefficients are potentially corrupted by the innate difficulty of measurement on the one hand, and their context of measurement being two-, rather than three-, dimensional on the other. It is possible that a solution to a continuous diffusion equation could be used instead of the present discretized numerical procedure, although our attempts at this method gave rise to either very unrealistic or computationally costly outcomes.

Acknowledgements. The work on this paper was supported by a Marie Curie European Reintegration Grant within the 7th European Community Framework Programme, grant agreement No. PERG03-GA-2008230993, by Polish Ministry of Science and Higher Education, grant No. N N201 362536 and by the Monash University Europe Travel Grant 2008/09 scheme. 


\section{References}

[1] J. J. Casciari, S. V. Sotirchos and R. M. Sutherland, Glucose diffusivity in multicellular tumor spheroids, Cancer Res. 48 (1988), 3905-3909.

[2] C. Crone and D. G. Levitt, Capillary permeability to small solutes, in: Handbook of Physiology: A critical, comprehensive presentation of physiological knowledge and concepts, Section 2: The Cardiovascular System, Vol. IV: Microcirculation, Part 1, E. M. Renkin and C. C. Michel (eds.), Amer. Physiol. Soc., Bethesda, 1984, 414 and $434-437$.

[3] J. P. Freyer, Role of necrosis in regulating the growth saturation of multi-cellular spheroids, Cancer Res. 48 (1988), 2432-2439.

[4] J. P. Freyer and R. M. Sutherland, Selective dissociation and characterization of cells from different regions of multicell tumour spheroids, ibid. 40 (1980), 3956-3965.

[5] - - - A reduction in the in situ rates of oxygen and glucose consumption of cells in emt6/ro spheroids during growth, J. Cell. Phys. 124 (1985), 516-524.

[6] - - - Regulation of growth saturation and development of necrosis in EMT6/Ro multicellular spheroids by the glucose and oxygen supply, Cancer Res. 46 (1986), 3504-3512.

[7] —, - , Proliferative and clonogenic heterogeneity of cells from EMT6/Ro multicellular spheroids induced by glucose and oxygen supply, ibid. 46 (1986), 3513-3520.

[8] P. Gerlee and A. R. A. Anderson, An evolutionary hybrid cellular automaton model of solid tumour growth, J. Theor. Biol. 246 (2007), 583-603.

[9] Y. Jiang, J. Pjesivac-Grbovic, C. Cantrell and J. Freyer, A multiscale model for avascular tumor growth, Biophys. J. 89 (2005), 3884-3894.

[10] A. R. Kansal, S. Torquato, E. A. Chioccaeb and T. S. Deisboeck, Emergence of a subpopulation in a computational model of tumor growth, J. Theor. Biol. 207 (2000), 431-441.

[11] A. R. Kansal, S. Torquato, G. R. Harsh Iva, E. A. Chioccaeb and T. S. Deisboeck, Simulated brain tumor growth dynamics using a three-dimensional cellular automaton, J. Theor. Biol. 203 (2000), 367-382.

[12] Y. Mansury and T. S. Deisboeck, The impact of 'search precision' in an agent-based tumour model, ibid. 224 (2003), 325-337.

[13] W. Mueller-Klieser, J. P. Freyer and R. M. Sutherland, Evidence for a major role of glucose in controlling development of necrosis in EMT6/Ro multicell tumor spheroids, Adv. Exp. Med. Biol. 159 (1983), 487-495.

[14] B. Müller, M. Riedel and P. J. Thurner, Three-dimensional characterization of cell clusters using synchrotron-radiation-based micro-computed tomography, Microsc. Microanal. 12 (2006), 97-105.

[15] A. T. Mustafin and E. I. Volkov, On the distribution of cell cycle generation times, Biosystems 15 (1982), 111-126.

[16] A. A. Patel, E. T. Gawlinski, S. K. Lemieux and R. A. Gatenby, A cellular automaton model of early tumor growth and invasion: The effects of native tissue vascularity and increased anaerobic tumour metabolism, J. Theor. Biol. 213 (2001), 315-331.

[17] M. J. Piotrowska and S. D. Angus, A quantitative cellular automaton model of in vitro multicellular spheroid tumour growth, ibid. 258 (2009), 165-178.

[18] - - - Corrigendum to "A quantitative cellular automaton model of in vitro multicellular spheroid tumour growth", ibid. (2009), available online at: http://dx.doi.org/ 10.1016/j.jtbi.2009.05.020 
[19] H. D. Preisler, A. Raza, V. Gopal, S. Ahmad and J. Bokhari, Distribution of cell cycle times amongst the leukemia cells within individual patients with acute myelogenous leukemia, Leukemia Res. 19 (1995), 693-698.

[20] R. Venkatasubramanian, M. A. Henson and N. S. Forbes, Incorporating energy metabolism into a growth model of multicellular tumor spheroids, J. Theor. Biol. 242 (2006), 440-453.

[21] S. Walenta, J. Doetsch, W. Mueller-Klieser and L. A. Kunz-Schughart, Metabolic imaging in multicellular spheroids of oncogene-transfected fibroblasts, J. Histochem. Cytochem. 48 (2000), 509-522.

[22] E. I. Zacharaki, G. S. Stamatakos, K. S. Nikita, N. K. Uzunoglu, Simulating growth dynamics and radiation response of avascular tumour spheroidsmodel validation in the case of an EMT6/Ro multicellular spheroid, Computer Methods and Programs in Biomedicine 6 (2004), 193-206.

[23] L. Zhang, C. G. Strouthos, Z. Wang and T. S. Deisboeck, Simulating brain tumor heterogeneity with a multiscale agent-based model: linking cell signaling, motility bias and expansion rate, preprint, http://arxiv.org/vc/q-bio/papers/0612/0612037v1.pdf, 2007.

Simon D. Angus

Department of Economics

Monash University

Clayton 3800, Australia

E-mail: simon.angus@buseco.monash.edu.au
Monika J. Piotrowska Department of Mathematics, Informatics and Mechanics University of Warsaw Banacha 2 02-097 Warszawa, Poland E-mail: monika@mimuw.edu.pl

Received on 12.11.2008;

revised version on 24.8.2009 